\title{
Preparation and Switching Behavior Characterization of Some Quaternary Thallium Chalcogenide Compounds
}

\author{
R.H. Al ORAINY* AND A.T. NAGAT \\ Physics Department, Faculty of Science for Girls, King Abdulaziz University, Jeddah, Saudi Arabia \\ (Received April 22, 2012; in final form October 20, 2012)
}

Investigation of the switching phenomenon on TlGaSSe single crystal revealed that it is typical for a memory switch. The switching process takes place with both polarities on the crystal and have symmetrical shapes. Current-voltage characteristics (CVC) of symmetrical Ag/TlGaSSe/Ag structures exhibit two distinct regions, high resistance "OFF" state and low resistance "ON" state having negative differential resistance. In addition, TlGaSSe is a quarternary semiconductor exhibiting $S$-type $I-V$ characteristics. The experimental results indicate that the phenomenon in our sample is very sensitive to temperature; light intensity and sample thickness. The switching parameters were checked under the influence of different factors of the ambient condition. The present investigation is the first one on switching phenomenon of TlGaSSe.

DOI: 10.12693/APhysPolA.123.121

PACS: $74.25 . \mathrm{fc}$

\section{Introduction}

Electrical threshold switching and memory in semiconductors have aroused great interest in recent years. Among the materials in which these effects take place there are chalcogenides. Some of the ternary and quaternary compounds are known to have nonlinear currentvoltage $(I-V)$ characteristics and switch from the high resistance "OFF" state for lower applied voltages, to the low resistance "ON" state for higher voltages [1]. Hence, researchers are trying to discover new materials and compounds which can be used for operation as bistable devices. The quaternary layered TlGaSSe crystal belongs to the new group of layered semiconductors. This crystal is formed from $\mathrm{TlGaS}_{2}$ and $\mathrm{TlGaSe}_{2}$ crystals by replacing half of sulfur (selenium) atoms with selenium (sulfur) atoms. The crystal lattice has two-dimensional layers arranged parallel to the (001) plane $[2,3]$. The presence of an $S$-type $I-V$ characteristic in the $\mathrm{TlGaS}_{2}$ [4] and the $\mathrm{TlGaSe}_{2}[5]$ has already been confirmed, and a further investigation of the electrical properties of TlGaSSe will be necessary. This aim is supported by the fact that there are fewer references concerning $S$-type electrothermal mechanism in single crystals than about amorphous chalcogenide systems. The switching itself could play an important role in technological applications (switching and memory devices, thermistors). Some of the physical properties of the TlGaSSe have been investigated by many authors [6-10].

The aim of this work is to study the switching phenomenon in thallium gallium sulphur selenite single crystal in wide range of temperatures as well as light intensity and sample thickness. To the best of our knowledge switching investigation in this compound has not been reported yet. The absence of data on the characteristics of switching about the compound TlGaSSe makes it dif-

*corresponding author; e-mail: r-aloraini@hotmail.com ficult to estimate potential application of this material in electronic technology.

\section{Experimental arrangement \\ 2.1. Sample preparation}

The modified Bridgman method was used to grow TlGaSSe layered single crystal from a stoichiometric melt of starting materials sealed in evacuated $\left(\approx 10^{-6}\right.$ mbar $)$ and carbon coated quartz ampoules with a tip at the bottom. All the starting materials used were of extra pure elements (99.9999\%). To prevent the ampoule from exploding, it was heated in a temperature gradient furnace, so that the sulphur condensed at the cold end and slowly reacted with the heated elements at the hot end. The ampoule was kept at temperature higher than the melting point for $10 \mathrm{~h}$ to ensure homogenization. The growth was achieved by lowering the ampoule from the hot side of the furnace at $850^{\circ} \mathrm{C}$, to the cold side at $480^{\circ} \mathrm{C}$ at a rate of $1.2 \mathrm{~mm} \mathrm{~h}^{-1}$. Details of the experimental procedure for crystal growth were reported elsewhere [11]. The resulting ingots had no cracks and voids on the surface. The crystals obtained have a layered structure (red in color) showed good optical quality and the freshly cleaved surfaces were mirror-like. The samples were identified by means of X-ray analysis. The X-ray diffraction analysis confirmed that TlGaSSe compound has monoclinic structure with lattice parameters $a=7.58 \AA, b=7.65 \AA$, $c=8.71 \AA$, and $\beta=111.85^{\circ}$. All samples of TlGaSSe used in the present study were freshly cleaved from crystals grown in the same ampoule. They had parallel faces and thickness of the order of $1.8 \mathrm{~mm}$.

\subsection{Switching effect measurements}

Specimens of TlGaSSe with plane-parallel mirror surfaces were prepared from a large ingot; they were properly cleaved along the cleavage plane and cut with a lancet. Their typical dimensions are $6.4 \times 3.5 \times 1.8 \mathrm{~mm}^{3}$. The samples are symmetric sandwich type structure in 
which single crystal samples are placed between two metal electrodes. The sample with its holder was positioned in a special system to allow temperature control in the investigated range. Details of the sample holder were described early [12]. The system was attached to a vacuum pump giving the possibility of measurements under vacuum. The environment temperature of the specimen under test was measured by means of a calibrated spot-welded chromel-alumel thermocouple. The investigation was carried out in a wide range of temperature in order to show the influence of ambient temperature on switching behavior. The current-voltage characteristic was measured using DC stabilized and regulated voltage supplied by means of digital programmable power supply model thermo EC 6000P. The current was measured by means of digital Keithley 617 electrometer. The current passing through the sample can easily be reversed or cut-off by applying three-pole double stage reversing switch. In order to investigate the effect of light intensity on the switching phenomena at $300 \mathrm{~K}$, samples with appropriate thickness were mounted in a cryostat equipped with suitable windows and clamped in its holder provided with apertures to allow the passage of the radiation. Details of the apparatus and cryostat as well as the used circuit are described in Ref. [13]. The sample was illuminated at normal incidence. Luxmeter (the Fisher scientific mark) was used for measuring light intensity. The current and the potential drop across the sample as a function of intensity of illumination were registered directly. The effect of sample thickness on the CVC was also studied. The specimen with initial thickness equal to $1.8 \mathrm{~mm}$ was first tested for the current-voltage characteristic and then its thickness was successively reduced. Samples with thickness varying in the range $1.8-0.8 \mathrm{~mm}$ were used to investigate the influence of the sample thickness on the switching characteristics. The overall accuracy of the measurements was estimated at $\pm 1 \%$.

\section{Results and discussion}

\subsection{Current-controlled negative resistance with memory}

In the present work we investigated the switching phenomenon when the current flows parallel to the cleavage planes perpendicular to the $c$-axis.

Figure 1 shows the $I-V$ characteristic of TlGaSSe single crystal, where it can be seen that initially in high resistance (OFF state) the voltage across the sample varies ohmically with current. Near a critical voltage $V_{\text {th }}$ (corresponding to a critical current $I_{\mathrm{th}}$ ), the characteristic becomes nonlinear. At $V_{\text {th }}$ the sample exhibits a negative resistance behavior which leads to a low-resistance $\mathrm{ON}$ state region. In the $\mathrm{ON}$ state the $I-V$ characteristic is nearly linear and the dynamic resistance is almost zero. The general behavior of the current-voltage characteristics for virgin sample of TlGaSSe single crystal has characteristic shapes as given schematically in Fig. 1.

Switching is symmetric with respect to the polarity. Also we can notice that thallium gallium sulphur selenite is a quaternary semiconductor exhibiting $S$-type $I-V$

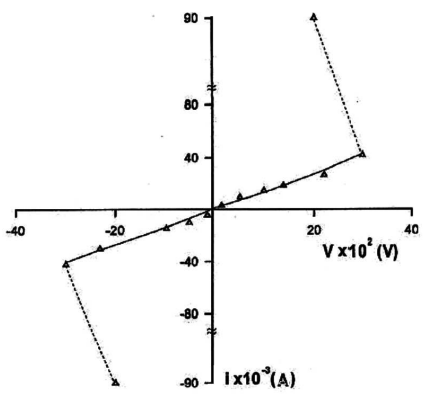

Fig. 1. Symmetrical of the CVC of TlGaSSe relative to the polarity.

characteristics, similar to the curves shown by many authors $[4,5,13-15]$. The sample remains in the ON-state if the current reduced to zero, i.e. under the zero bias condition, the sample stays in the ON-state. This indicates that the memory state persists if the current is decreased slowly to its zero value. However, if the current was forced to decay suddenly, the specimen returns to the high resistance state.

\subsection{Temperature dependence of switching phenomena of TlGaSSe crystal}

The temperature dependence of the $I-V$ characteristics is an important factor in considering a switching material for information storage applications. In the present study, the effect of temperatures on the current-voltage characteristic and switching behavior of TlGaSSe compound have been investigated in the temperature range extended from 150 to $330 \mathrm{~K}$. Usually, in memory switching materials, different samples of the same thickness are used to study the effect of temperature on the switching behavior. The temperature dependence of the $I-V$ characteristics for TlGaSSe compound is shown in Fig. 2.

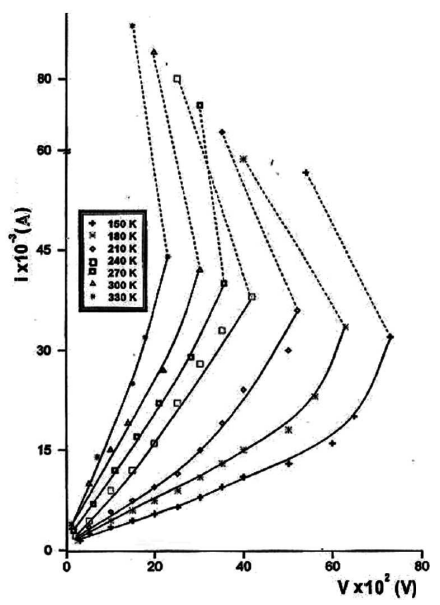

Fig. 2. Current-voltage characteristics at different values of temperature for TlGaSSe single crystal.

As is evident from the experimental curves as well as predicted by the electrothermal model [16-18], the ambient temperature greatly influences the form of the $I-V$ 
curves. As observed from the curves of Fig. 1 and Fig. 2 some common features are shown such as the following.

There are two distinct regions; one in the OFF state region and the other negative differential resistance (NDR) region.

It is clear that, with increase in temperature, the CVC as a whole is shifted toward the lower potentials.

$S$-shaped curves in the higher current density regions with a rather pronounced NDR region, which sets in after a critical current value $\left(I_{\mathrm{th}}\right)$, threshold current.

The NDR region of the curves is more pronounced at higher ambient temperature.

The transition from the low to the high conductivity state of the curves is almost abrupt at higher temperature.

The threshold voltage $V_{\mathrm{th}}$, after which the NDR region sets in, becomes higher with decreasing temperature.

A marked increase in the holding current $I_{\mathrm{h}}$ with increase in temperature, while the holding voltage gradually increases with decrease in temperature. The effect of the ambient temperature on the switching parameters $V_{\mathrm{th}}$ and $I_{\mathrm{th}}$ is illustrated in Fig. 3.

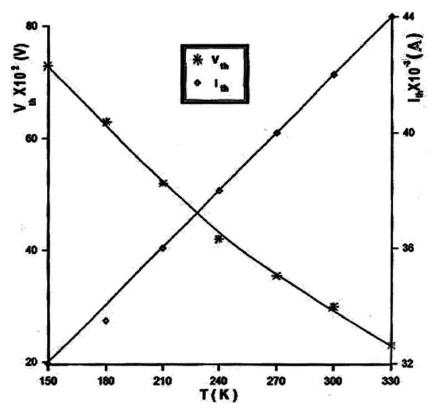

Fig. 3. Ambient temperature effect on threshold current and voltage for TlGaSSe single crystal.

It is clear from the figure that as the temperature increases, the threshold voltage decreases and the threshold current increases. This indicates that an electrothermal mechanism is involved in the switching process. The dependence of $v_{\mathrm{th}}^{1 / 2}$ and $P_{\mathrm{th}}$ on $T$ is plotted in Fig. 4 on the basis of the thermal-field Frenkel effect [19].

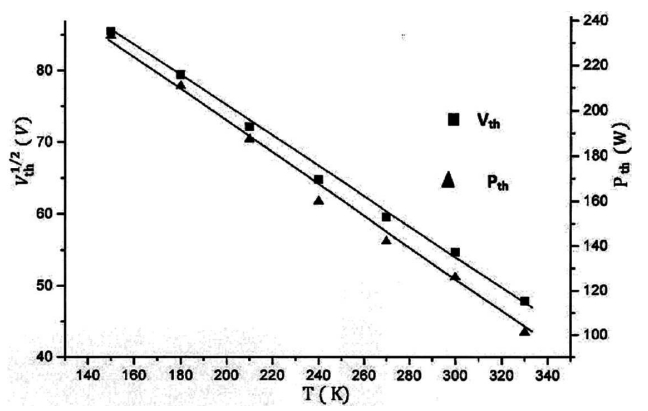

Fig. 4. Temperature dependence of $v_{\mathrm{th}}^{1 / 2}$ and $P_{\mathrm{th}}$ for TlGaSSe single crystal.
This figure obeys the following relation [19]:

$$
V_{\mathrm{th}}^{1 / 2}=\left(\frac{\pi \varepsilon_{0} \varepsilon_{\infty} d}{e}\right)^{1 / 2}(\varphi-c T),
$$

where $\varepsilon_{0}$ is the permittivity of vacuum, $\varepsilon_{\infty}$ is the electron component of the permittivity, $d$ is the distance between two electrodes, $c$ is a constant, $e$ is the electron charge, $\varphi$ is the depth of the potential well, $T$ is the absolute temperature. Using the coordinates $V_{\text {th }}^{1 / 2}$ and $T$ clearly, within the experimental error, the result is fitted by a straight line. This shows that the switching in $\mathrm{Ag} / \mathrm{TlGaSSe} / \mathrm{Ag}$ structures from high to low resistivity state occurs under the simultaneous action of an electric field and temperature [20]. This must be supported by the dependence of threshold field on the thickness of the active region. The power necessary to change the material from the high-resistance state to the low-resistance state called threshold power $\left(P_{\text {th }}\right)$. The threshold power depends also on the ambient temperature.

The dependence of the threshold power on temperatures was plotted in Fig. 4 . It is found that $P_{\text {th }}$ decreases linearly with temperature. So this result is quite logical, since the power necessary to initiate switching decreases when temperature increases. This explains why at low temperature large switching power is required. The OFF and $\mathrm{ON}$ state resistance ratio $\left(R_{\mathrm{OFF}} / R_{\mathrm{ON}}\right)$ also depends on temperature. The dependence of the resistance ratio on temperature shows that $\left(R_{\mathrm{OFF}} / R_{\mathrm{ON}}\right)$ increases with temperature indicate that the surrounding temperature affects the sample resistance ratio. The ON-state resistance is typically $22.22 \times 10^{3} \Omega$ at room temperature and depends on the magnitude of the threshold voltage, as $V_{\mathrm{th}}$ decreases, $R_{\mathrm{ON}}$ decreases. The resistance ratio $\left(R_{\mathrm{OFF}} / R_{\mathrm{ON}}\right)$ for our samples at room temperature is of the order of 1.9 .

\subsection{Influence of light intensity on switching effect}

The current-voltage characteristics at different values of light intensity at room temperature for TlGaSSe are plotted in Fig. 5.

The switching phenomenon of our sample was very sensitive to the light intensity. The CVC under illumination $(20,400,800,1200,1600,2000 \mathrm{~lx})$ has the general form of switching with $S$-shape. It is evident from this figure that the $I-V$ characteristics as a whole are shifted toward lower potentials with an increase in the intensity of the incident light, also we can see that the form of CVC and the magnitude of the photocurrent depend strongly on the intensity of the incident light. This means that in case of weak illumination the threshold voltage is larger and the threshold current value is smaller than the value obtained in case of intense light. Figure 6 illustrates the dependence of $V_{\mathrm{th}}$ and $I_{\mathrm{th}}$ on light intensity.

As can be seen, threshold voltage decreases with increasing light intensity, whereas the threshold current increases with light illumination. The main contribution comes from photocarrier generation through excitation states and is larger than recombination process. The re- 


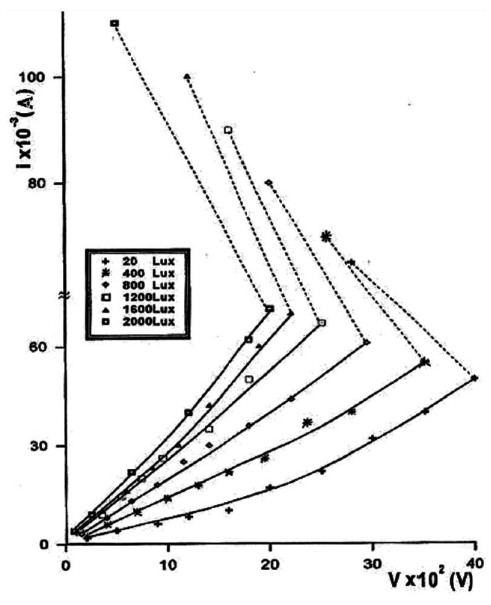

Fig. 5. The effect of light intensity on $I-V$ characteristics of TlGaSSe specimen.

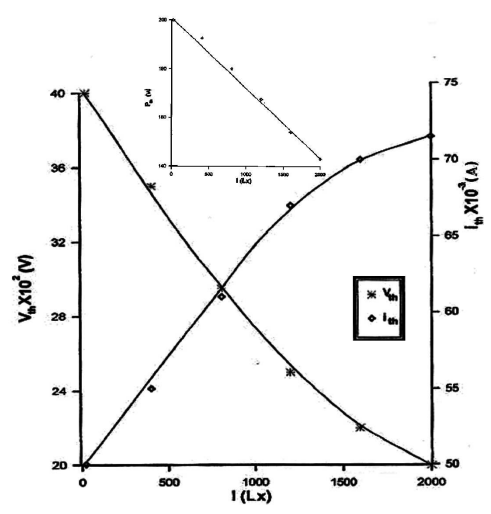

Fig. 6. Dependence of $I_{\mathrm{th}}$ and $V_{\mathrm{th}}$ on light illumination for TlGaSSe compound. Effect of light intensity on threshold power $P_{\text {th }}$.

lation between threshold power $P_{\text {th }}$ with light intensity is presented graphically in figure.

As we notice, $P_{\mathrm{th}}$ decreases linearly with increase of the incident light intensity. This may be due to photogeneration processes which took place under illumination of the sample and led to low power for switching as the intensity dose increases. The dependence of the resistance ratio $R_{\mathrm{OFF}} / R_{\mathrm{ON}}$ on the illumination intensity was also determined. This ratio increases gradually as the light intensity increases in the low intensity region while it increases rapidly with light illumination in the high intensity region. The $\mathrm{ON}-$ state resistance at room temperature as calculated is 2.0 times smaller than the OFF-state at $20 \mathrm{~lx}$ and decreases as the light intensity increases becoming 0.14 times smaller than the OFF-state at $2000 \mathrm{~lx}$.

\subsection{Effect of sample thickness}

The overall features of the $I-V$ characteristics of TlGaSSe are altered by changes in sample thickness. It

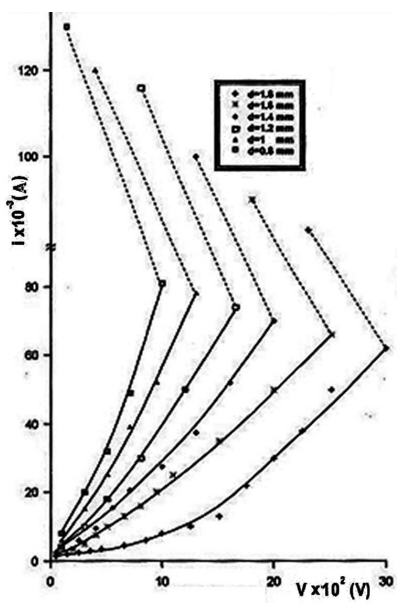

Fig. 7. The effect of the specimen thickness on switching phenomena for TlGaSSe.

is important to observe the influence of the sample thickness on the switching characteristics. Room temperature $I-V$ characteristics for the investigated compound were studied for samples of thickness $1.8-0.8 \mathrm{~mm}$. Figure 7 represents the dependence of the switching behavior on the thickness of TlGaSSe specimen.

The figure indicates that the threshold potential changes with the specimen thickness and the width of the dashed lines, which represent the variation from the OFF to ON state, decreases with increase of thickness. This result indicates that the switching can be easily controlled with the sample thickness. The holding current and voltage are also affected with the active thickness of the specimen. A variation of the threshold voltage and current with thickness sample can be observed from Fig. 8 which represents a graph of $V_{\mathrm{th}}$ and $I_{\mathrm{th}}$ against $d$. It is clear from the curves that the threshold voltage decreases exponentially with decrease of the sample thickness, while the threshold current increases linearly with decrease of sample thickness. One can say that the decrease of the specimen thickness lowers the potential for the switching process in a specimen. This result can be explained clearly in terms of the Ovshinsky model [21] in which injection and impact ionization are believed to play an important roles in such process.

Figure 9 shows that the dependence of the threshold field $E_{\text {th }}$ and threshold power $P_{\text {th }}$ on the thickness of the sample.

It is clear that $E_{\text {th }}$ increases with sample thickness. This indicates that the electric field has a profound influence on the ability of the samples to undergo a transition from the OFF state to an effective region of NDR. This supports the suggestion that the mechanism of the switching in TlGaSSe sample may involve both electronic and thermal processes [22]. The variation of the threshold power $P_{\text {th }}$ with sample thickness is plotted in the same figure.

It is seen that the threshold power increases linearly with increasing thickness, i.e. the power required for 


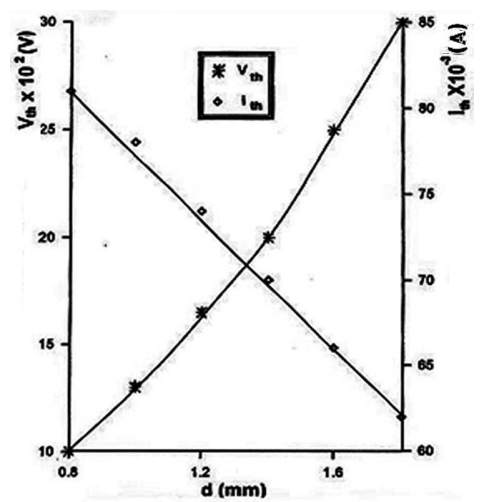

Fig. 8. Variation of $V_{\text {th }}$ and $I_{\text {th }}$ with TlGaSSe sample thickness.

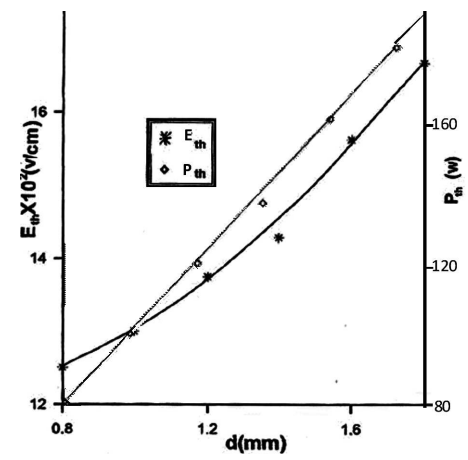

Fig. 9. The dependence of the threshold field $E_{\text {th }}$ and threshold power $P_{\text {th }}$ on the thickness of TlGaSSe sample.

switching decreases as the thickness of the sample decreases. The ratio between $R_{\mathrm{OFF}}$ and $R_{\mathrm{ON}}$ state resistivities decreases with thickness and reaches a very low value at higher thickness. The resistance ratio varies from 1.7 to 10.7 in the range of sample thickness under test. Increasing the thickness leads to smaller value of this ratio. These results indicate that the switching process can be easily controlled with specimen thickness.

\section{Conclusion}

In view of the possibility of the practical application of the switching effect with memory, we have grown and studied single crystal of TlGaSSe. In conclusion, first observations of the switching process in TlGaSSe layered chalcogenide semiconductor crystals are reported. Current-controlled negative resistance (CCNR) in TlGaSSe single crystal has been reported for the first time. It has been found that thallium gallium sulphur selenite single crystals exhibit bistable or memory switching. The current-voltage characteristics are symmetrical with respect to the reverse of the applied voltage and current. The results strongly indicated that the phenomenon in our sample is very sensitive to temperature, light intensity, and sample thickness. The switching parameters were checked under the influence of different factors of the ambient condition. The TlGaSSe with such properties can be used as switching and memory elements in modern electronic devices.

\section{Acknowledgments}

This paper was funded by the Deanship of Scientific Research (DSR), King Abdulaziz University, Jeddah, under grant No. 7-843-D1432. The authors, therefore, acknowledge with thanks DSR technical and financial support.

\section{References}

[1] V.D. Das, K.S. Raju, A. Bhaskaran, J. Appl. Phys. 78, 3262 (1995).

[2] A.M Panich, J. Phys. Condens. Matter 20, 293202 (2008).

[3] K.A. Yee, A. Albright, J. Am. Chem. Soc. 113, 6474 (1981).

[4] A.A. AlGhamdi, A.T. Nagat, F.S. Bahabri, R.H. AlOrainy, S.E. Al Garni, Appl. Surf. Sci. 257, 3205 (2011)

[5] S.A. Hussein, G. Attia, S.R. Al-Harbi, A.A. AlGhamdi, F.S. AlHazmi, S.E. Al-Garni, J. King Abdulaziz Univ. Sci. 21, 27 (2009).

[6] N.M. Gasanly, J. Alloys Comp. 509, 4205 (2011).

[7] I. Guler, N.M. Gasanly, J. Korean Phys. Soc. 51, 2031 (2007)

[8] K.R. Allakhverdiev, Solid State Commun. 111, 253 (1999)

[9] I. Kucuk, T. Yildirim, N.M. Gasanly, H. Ozkan, Expert Syst. Appl. 38, 7192 (2011)

[10] T. Yildirim, N.M. Gasanly, Curr. Appl. Phys. 9, 1278 (2009)

[11] S.A. Hussein, A.T. Nagat, Cryst. Res. Technol. 24, 283 (1989)

[12] A.T. Nagat, S.A. Hussein, Y.H. Gameel, G.A. Gamal, Phys. Status Solidi A 121, 201 (1990).

[13] A.A. AlGhamdi, S.A. Hussein, M.M. Nassary, Mater. Sci. Res. India 2, 107 (2004).

[14] A.A. AlGhamdi, A.T. Nagat, F.S. Bahabri, Hazmi, J. Alloys Comp. 484, 561 (2009).

[15] R.H. AlOrainy, Acta Phys. Pol. A 121, 666 (2012).

[16] K.W. Boer, S.R. Ovshinsky, J. Appl. Phys. 41, 2675 (1970)

[17] G.C. Vezzoli, P.J. Walsh, L.W. Doremus, J. Non-Cryst. Solids 18, 333 (1975)

[18] T. Nagat, F.S. Bahabri, M.M. Mobark, H.T. Shaban, A.A. Al Ghamdi, S.R. Alharbi, King Abdulaziz Univ. Sci. 20,39 (2008)

[19] S.I. Aliev, G.M. Niftiev, F.I. Pliev, B.G. Tagiev, Sov Phys. Semicond. 13, 340 (1979).

[20] V.G. Kolomiets, E.A. Lebedev, I.A. Taksmi, Sov. Phys. Semicond. 3, 267 (1969).

[21] S.R. Ovshinsky, Phys. Rev. Lett. 21, 1450 (1968),

[22] S. Prakash, S. Asokan, D.B. Ghare, J. Phys. D, Appl. Phys. 29, 2004 (1996) 